PROCEEDINGS OF THE

AMERICAN MATHEMATICAL SOCIETY

Volume 132, Number 1, Pages 7-14

S 0002-9939(03)07214-9

Article electronically published on August 12, 2003

\title{
BROUÉ'S ABELIAN DEFECT GROUP CONJECTURE FOR ALTERNATING GROUPS
}

\author{
ANDREI MARCUS
}

(Communicated by Stephen D. Smith)

\begin{abstract}
We establish Broué's abelian defect group conjecture for the alternating groups, using the Chuang-Rouquier theorem (proving this for the symmetric groups) and a descent result.
\end{abstract}

\section{INTRODUCTION}

A conjecture of M. Broué states that a block algebra of a finite group is derived equivalent to its Brauer correspondent if the defect group of the block is abelian. We establish this conjecture for the alternating groups, based on the Chuang-Rouquier theorem (proving this for the symmetric groups) and on a descent result coming from Clifford theory.

To be more precise, let $G=A_{n}, \mathcal{O}$ a complete discrete valuation ring with algebraically closed residue field $k$ of characteristic $p>0$, let $b$ be a block of $\mathcal{O} G$ with defect group $D$, and let $c$ be the Brauer correspondent block of $\mathcal{O} N_{G}(D)$.

We show that if $D$ is abelian, then the algebras $A=b \mathcal{O} G$ and $B=c \mathcal{O} N_{G}(D)$ are splendidly derived equivalent, that is, there is a bounded complex $X$ of $(A, B)$ bimodules such that its components are $p$-permutation modules whose indecomposable summands have vertices contained in $\delta(D)=\{(u, u) \mid u \in D\}$, and such that $X \otimes_{B} X^{\vee} \simeq A$ in the homotopy category of complexes of $(A, A)$-bimodules, and $X^{\vee} \otimes_{A} X \simeq B$ in the homotopy category of $(B, B)$-bimodules, where $X^{\vee}$ denotes the $\mathcal{O}$-dual of $X$.

Moreover, we show that there is such an equivalence that is compatible with $p^{\prime}$-outer automorphism groups, which means in our case the existence of a tilting complex having an $\operatorname{Aut}(G) / G$-grading. The complete statement of the main result is given in Theorem 3.1 below. This additional condition is especially important in the case of principal blocks, where it is used to reduce the conjecture to the case of simple groups.

In our proof, we use that the conjecture is known to hold for the symmetric group $S_{n}$ by the work of J. Rickard, J. Chuang, R. Kessar and R. Rouquier, and we show how to "go down" to $A_{n}$. Important inspiration comes from the paper [5] of P. Fong and M. Harris, who verified the weaker "isotypy form" of the conjecture for $A_{n}$, by

Received by the editors August 15, 2002.

2000 Mathematics Subject Classification. Primary 20C20; Secondary 20C30, 16W50.

Key words and phrases. Symmetric groups, alternating groups, blocks, abelian defect groups, Rickard equivalences, splendid tilting complex, group graded algebras.

The author was supported by a Fulbright fellowship. 
using Rouquier's paper [11] on $S_{n}$. Note that a similar procedure was devised by E. Dade in [4] leading to the verification of his Invariant Projective Conjecture for $A_{n}$, and we are able to adapt some techniques from there.

In Section 2 we consider $\mathcal{O}$-algebras graded by the cyclic group $C_{n}$ of order $n$ not divisible by $p$. The main technical result is that a bimodule over two such algebras is $C_{n}$-graded if and only if the group $\hat{C}_{n}$ of linear characters of $C_{n}$ acts on it. If a complex $X$ induces a Rickard equivalence between two strongly $C_{n}$-graded algebras $R$ and $S$, then we obtain a Rickard equivalence between the 1 -components $R_{1}$ and $S_{1}$ provided that $X$ is a complex of $C_{n}$-graded bimodules.

We discuss blocks of symmetric and alternating groups in Section 3, where we prove Theorem [3.1. The interesting case in when the block $b$ of $\mathcal{O} S_{n}$ is selfassociated, that is, it is invariant under the action of $\hat{C}_{2}$. Then the algebra $b \mathcal{O} S_{n}$ is $C_{2}$-graded, and so is its Brauer correspondent $c \mathcal{O} N_{S_{n}}(D)$; we only need to show that the terms of the complex inducing a Rickard equivalence between these blocks are also $C_{2}$-graded.

We refer the reader to [13] for equivalences between blocks, and to [7] for equivalences induced by graded bimodules. All the facts on the representation theory of symmetric groups that we use here are contained in [2] and [5].

\section{Algebras graded By a CYClic group}

2.1. Let $C_{n}=\langle\sigma\rangle$ be the cyclic group of order $n$, and let $(\mathcal{K}, \mathcal{O}, k)$ be a $p$-modular system, where $p$ does not divide $n$, such that $\mathcal{K}$ contains a primitive $n$-th root $\epsilon$ of unity. The group

$$
\hat{C}_{n}=\operatorname{Hom}\left(C_{n}, \mathcal{K}^{\times}\right)
$$

of characters of $C_{n}$ is isomorphic to $C_{n}$, and we have that $\hat{C}_{n}=\langle\hat{\sigma}\rangle$, where $\hat{\sigma}(\sigma)=\epsilon$. 2.2. Let $R=\bigoplus_{g \in C_{n}} R_{g}$ be a $C_{n}$-graded $\mathcal{O}$-algebra, not necessarily strongly graded. Then $\hat{C}_{n}$ acts on $R$ as automorphisms of $C_{n}$-graded algebras by

$$
\hat{\rho}^{\hat{\rho}_{g}}=\hat{\rho}(g) r_{g}
$$

for all $g \in C_{n}, \hat{\rho} \in \hat{C}_{n}$, and the components of $R$ can be recovered as

$$
R_{\sigma^{j}}=\left\{\left.r \in R\right|^{\hat{\sigma}} r=\epsilon^{j} r\right\},
$$

for $j=0, \ldots, n-1$. We may form the skew group algebra

$$
R * \hat{C}_{n}=\left\{r \hat{\rho} \mid r \in R, \hat{\rho} \in \hat{C}_{n}\right\},
$$

with multiplication defined by

$$
(r \hat{\rho})\left(r^{\prime} \hat{\rho}^{\prime}\right)=r \cdot \hat{\rho} r^{\prime} \cdot \hat{\rho} \hat{\rho}^{\prime} .
$$

Proposition 2.3. The category $R$-Gr of $C_{n}$-graded (left) $R$-modules is isomorphic to the category $R * \hat{C}_{n}$-Mod.

Proof. Let $M=\bigoplus_{g \in C_{n}} M_{g}$ be a $C_{n}$-graded $R$-module. Then $M$ becomes an $R * \hat{C}_{n^{-}}$ module with multiplication defined by

$$
(r \hat{\rho}) m_{g}=\hat{\rho}(g) r m_{g},
$$

for all $r \in R, g \in C_{n}, m_{g} \in M_{g}$ and $\hat{\rho} \in \hat{C}_{n}$. If $f: M \rightarrow M^{\prime}$ is a map of $C_{n}$-graded $R$-modules, then one easily verifies that $f$ is also $R * \hat{C}_{n}$-linear. 
Conversely, let $M$ be an $R * \hat{C}_{n}$-module, and let

$$
M_{\sigma^{j}}=\left\{m \in M \mid \hat{\sigma} m=\epsilon^{j} m\right\} .
$$

Then every element $m \in M$ is written uniquely as $m=\sum_{j=0}^{n-1} m_{\sigma^{j}}$, with $m_{\sigma^{j}} \in M_{\sigma^{j}}$ for $j=0, \ldots, n-1$, where

$$
m_{\sigma^{j}}=n^{-1} \sum_{j=0}^{n-1}\left(\epsilon^{-1} \sigma\right)^{j} m
$$

Again, it is routine to verify that $R_{g} M_{h} \subseteq M_{g h}$ for all $g, h \in C_{n}$, and if $f: M \rightarrow M^{\prime}$ is a map of $R * \hat{C}_{n}$-modules, then $f\left(M_{g}\right) \subseteq M_{g}^{\prime}$ for all $g \in C_{n}$.

2.4. Let $R$ and $S$ be two $C_{n}$-graded $\mathcal{O}$-algebras. Then $\hat{C}_{n}$ acts on $R \otimes_{\mathcal{O}} S^{\text {op }}$ diagonally by

$$
\hat{\rho}(r \otimes s)=\hat{\rho}_{r} \otimes \hat{\rho}^{-1} s,
$$

for all $\hat{\rho} \in \hat{C}_{n}, r \in R$ and $s \in S$. So we may consider the skew group algebra $\left(R \otimes_{\mathcal{O}} S^{\mathrm{op}}\right) * \hat{C}_{n}$. As in Proposition 2.3 , one can prove that the category $R$-Gr- $S$ of $C_{n}$-graded $(R, S)$-bimodules is isomorphic to the category $\left(R \otimes_{\mathcal{O}} S^{o p}\right) * \hat{C}_{n}$-Mod.

If $M$ is an $(R, S)$-bimodule and $\hat{\rho} \in \hat{C}_{n}$, then the $\hat{\rho}$-th conjugate ${ }^{\hat{\rho}} M$ of $M$ is defined by

$$
{ }^{\hat{\rho}} M=\left(R \otimes_{\mathcal{O}} S^{\mathrm{op}}\right) \hat{\rho} \otimes_{R \otimes_{\mathcal{O}} S^{\mathrm{op}}} M .
$$

Observe that we obtain an isomorphic $(R, S)$-bimodule if we set ${ }^{\hat{\rho}} M=M$ as $\mathcal{O}$ modules, and multiplication

$$
(r \otimes s) \cdot \hat{\rho} m=\hat{\rho}^{-1}(r \otimes s) \cdot m,
$$

for all $m \in M, r \in R, s \in S$ and $\hat{\rho} \in \hat{C}_{n}$. It easily follows that if $T$ is another $C_{n}$-graded $\mathcal{O}$-algebra and $N$ is an $(S, T)$-bimodule, then for all $\hat{\rho} \in \hat{C}_{n}$ there is an isomorphism

$$
\hat{\rho}(M \otimes N) \simeq{ }^{\hat{\rho}} N \otimes_{S}{ }^{\hat{\rho}} N
$$

of $(R, T)$-bimodules.

2.5. We are going to apply the above constructions to obtain a descent theorem for Rickard equivalences, which can also be regarded as an analogue of [4, Theorem 12.2].

Let $G^{+}$be a normal subgroup of the finite group $G$, with $G / G^{+} \simeq C_{n}$. Let $b$ be a block of $\mathcal{O} G$ with defect group $D \leq G^{+}$, let $H=N_{G}(D), H^{+}=N_{G^{+}}(D)$, and let $c \in \mathcal{O} H$ be the Brauer correspondent of $b$. If $e$ is a block of $\mathcal{O} G^{+}$covered by $b$, then the Brauer correspondent $f \in \mathcal{O} H^{+}$of $e$ is covered by $c$, by the Harris-Knörr correspondence.

The group $\hat{C}_{n}$ acts on the blocks of $\mathcal{O} G$ and $\mathcal{O} H$, and for each $\hat{\rho} \in \hat{C}_{n}$, the Brauer correspondent of ${ }^{\hat{\rho}} b$ is ${ }^{\hat{\rho}} c$. We denote by $\hat{C}_{n, b}$ the stabilizer of $b$ under this action. The group $C_{n}$ acts by conjugation of the blocks of $\mathcal{O} G^{+}$and $\mathcal{O} H^{+}$, and for each $g \in C_{n}$, the Brauer correspondent of ${ }^{g} e$ is ${ }^{g} f$. Let $C_{n, e}$ denote the stabilizer of $e$ in $C_{n}$. Consider the central idempotent

$$
b^{+}=\sum_{\hat{\rho} \in\left[\hat{C}_{n} / \hat{C}_{n, b}\right]} \hat{\rho} b=\sum_{g \in\left[C_{n} / C_{n, e}\right]}{ }^{g} e
$$

of $\mathcal{O} G^{+}$, where $\left[C_{n} / C_{n, e}\right]$ denotes a full set of representatives for the left cosets of $C_{n, e}$ in $C_{n}$. The second equality follows by [4 Lemma 9.9]. Let $c^{+}$be the 
similarly defined central idempotent of $\mathcal{O H}^{+}$, and consider the strongly $C_{n}$-graded algebras $R=b^{+} \mathcal{O} G=\mathcal{O} G e \mathcal{O} G$ and $S=c^{+} \mathcal{O} H=\mathcal{O H e O H}$. Note that $R$ is Morita equivalent to $e \mathcal{O G e}$ and $S$ is Morita equivalent to $f \mathcal{O} H f$.

With this notation, we have the following result, which is more general than we actually use in the case of alternating groups.

Theorem 2.6. Let $X$ be a complex of $(b \mathcal{O} G, c \mathcal{O} H)$-bimodules inducing a Rickard equivalence between $b \mathcal{O} G$ and $c \mathcal{O} H$, and consider the complex

$$
Y=\bigoplus_{\hat{\rho} \in\left[\hat{C}_{n} / \hat{C}_{n, b}\right]}{ }^{\hat{\rho}} X
$$

of $(R, S)$-bimodules.

If $\hat{\rho} Y \simeq Y$ as complexes of $(R, S)$-bimodules for all $\hat{\rho} \in \hat{C}_{n}$, then the block algebras $\mathrm{eO} \mathrm{O}^{+}$and $\mathrm{fOH}^{+}$are Rickard equivalent.

Proof. Since $\hat{C}_{n}$ is cyclic of order prime to $p$, the assumption implies, by [6], Proposition 3.11] and [13 Lemma 2.13], that $Y$ extends to a complex of $\left(R \otimes_{\mathcal{O}} S^{\mathrm{op}}\right) * \hat{C}_{n^{-}}$ modules. So by 2.4. $Y$ is a complex of $C_{n}$-graded $(R, S)$-bimodules. It follows by [7 Theorem 4.7] that the 1-component $Y_{1}$ of $Y$ is a Rickard tilting complex of $\left(R_{1}, S_{1}\right)$-bimodules. Hence $e Y_{1} f$ is a Rickard tilting complex of $\left(e \mathcal{O} G^{+}, f \mathcal{O H}^{+}\right)$bimodules.

\section{Blocks OF SYMmetric AND ALtERnATING GROUPS}

Let us state the main result of the paper.

Theorem 3.1. Let $p>2, G=S_{n}, G^{+}=A_{n}, \tilde{G}=\operatorname{Aut}\left(G^{+}\right), b^{+}$a block of $\mathcal{O} G^{+}$ with nontrivial abelian defect group $D, H^{+}=N_{G^{+}}(D)$, and $c^{+} \in \mathcal{O} H^{+}$the Brauer correspondent of $b^{+}$. Then there exists a splendid tilting complex of $\tilde{G} / G^{+}$-graded $\left(b^{+} \mathcal{O} \tilde{G}, c^{+} \mathcal{O} \tilde{H}\right)$-bimodules.

Actually, we only need to consider the case $p>2$. Indeed, if $p=2$, then by $[5$ Lemma (7.A)], $D \simeq C_{2} \times C_{2}$. In this case Broué's conjecture holds (even in the extended form) by [13, Section 6.3].

The proof of the theorem consists of several steps.

3.2. The block $b^{+}$is $C_{2}$-invariant. Let $b$ be a block of $\mathcal{O} G$ covering $b^{+}$and let $c \in \mathcal{O} H$ be the Brauer correspondent of $b$. We denote ${ }^{\hat{\sigma}} b=b^{*}$, where $C_{2}=\langle\hat{\sigma}\rangle$. If $b \neq b^{*}$, then $b \mathcal{O} G$ is isomorphic to $b^{+} \mathcal{O} G^{+}$and $c \mathcal{O H}$ is isomorphic to $c^{+} \mathcal{O} H^{+}$. Consequently, if $X$ is a splendid tilting complex of $(b \mathcal{O} G, c \mathcal{O} H)$-bimodules, then $X$ is also a splendid tilting complex of $\left(b^{+} \mathcal{O G}^{+}, c^{+} \mathcal{O} H^{+}\right)$-bimodules.

3.3. Assume that $b=b^{*}$, that is, $b$ is self-associated. Then $b=b^{+}, c=c^{*}=c^{+}$, and $b \mathcal{O} G$ and $c \mathcal{O H}$ are strongly $C_{2}$-graded algebras. We can apply Theorem 2.6 if we show that the splendid equivalence constructed in [2] and [3] is induced by a complex of $C_{2}$-graded bimodules. Since this equivalence is a composition of several equivalences, we shall examine the steps one by one.

Recall that the bloc $b$ corresponds uniquely to a $p$-core $\kappa$ and a $p$-weight $w<p$, and $D \simeq C_{p} \times \cdots \times C_{p}$ ( $w$ times). Write $n=p w+t$. Then, by [2, Section 3], the algebra $c \mathcal{O} H$ is isomorphic to $\mathcal{O} N_{S_{p w}}(D) \otimes_{\mathcal{O}} \mathcal{O} S_{t} c_{0}$, where $c_{0}$ is the block of defect zero of $\mathcal{O} S_{t}$ corresponding to the $p$-core $\kappa$. Recall also that since $b$ is self-associated, $\kappa$ is also self-associated, that is, its diagram is symmetric with respect to the main diagonal. Moreover, the block $\mathcal{O} N_{S_{p w}}(D)$ is isomorphic to $\mathcal{O}\left(\left(C_{p} \rtimes C_{p-1}\right) \curlywedge S_{w}\right)$. 
3.4. It was conjectured by R. Rouquier that there are blocks of weight $w$ of symmetric groups that are Morita equivalent to the principal block $B_{0}\left(S_{p} 2 S_{w}\right)$ of $\mathcal{O}\left(S_{p} \imath S_{w}\right)$. This conjecture was proved in [2, Section 4], where one of these blocks was defined as follows.

Consider an abacus having $w+i(w-1)$ beads on the $i$-th runner, $i=0,1, \ldots, p-1$, and let $\rho$ be the $p$-core having this abacus representation. Note that the core $\rho$ is self-associated.

Let $V$ be a set containing the disjoint union $U=U_{1} \cup \cdots \cup U_{w}$ of sets of cardinality $p$, and let $e$ be a block of $\mathcal{O} S(V)$ with defect group $D$ corresponding to the $p$-core $\rho$. Let $\tilde{N}$ be the subgroup of $S(U)$ consisting of permutations sending each $U_{i}$ to some $U_{j}$, let $N=\tilde{N} \times S(V \backslash U)$, and let $f \in \mathcal{O} N$ be the Brauer correspondent of $e$. Then the group $\tilde{N}$ is isomorphic to $S_{p} \prec S_{w}$, and the algebra $f \mathcal{O} N$ is isomorphic to $B_{0}\left(S_{p} \prec S_{w}\right) \otimes_{\mathcal{O}} \mathcal{O} S_{r} f_{0}$, where $f_{0}$ is the block of defect zero corresponding to the core $\rho$, and $r=|V \backslash U|$.

By [2] Theorem 2], the Green correspondent $M$ of $e \mathcal{O} S(V)$ with respect to $(S(V) \times S(V), S(V) \times N, \delta(D))$ induces a Morita equivalence between $e \mathcal{O} S(V)$ and $f \mathcal{O} N$. We claim that $M$ is a $C_{2}$-graded $(e \mathcal{O} S(V), f \mathcal{O} N)$-bimodule. Indeed, since

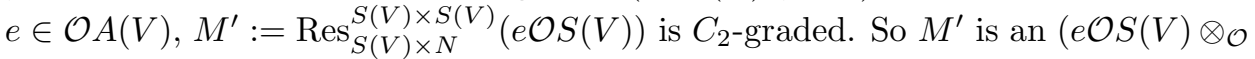
$\left.(f \mathcal{O} N)^{\mathrm{op}}\right) * \hat{C}_{2}$-module. Since $M$ is the unique direct summand of $M^{\prime}$ with vertex $\delta(D)$, it follows that $M$ is $\hat{C}_{2}$-invariant; hence $M$ is an $\left(e \mathcal{O} S(V) \otimes_{\mathcal{O}}(f \mathcal{O} N)^{\mathrm{op}}\right) * \hat{C}_{2^{-}}$ module. The claim now follows by 2.4

3.5. To see that there is a $C_{2}$-graded Rickard equivalence between $\mathcal{O}\left(\left(C_{p} \rtimes C_{p-1}\right)\right.$ ? $\left.S_{w}\right) \otimes_{\mathcal{O}} \mathcal{O} S_{t} c_{0}$ and $B_{0}\left(S_{p} \imath S_{w}\right) \otimes_{\mathcal{O}} \mathcal{O} S_{t} c_{0}$, note first that if $R=R_{1} \oplus R_{-1}$ and $S=S_{1} \oplus S_{-1}$ are $C_{2}$-graded algebras, then $R \otimes_{\mathcal{O}} S$ is $C_{2}$-graded, where

$$
\begin{aligned}
\left(R \otimes_{\mathcal{O}} S\right)_{1} & =R_{1} \otimes_{\mathcal{O}} S_{1} \oplus R_{-1} \otimes_{\mathcal{O}} S_{-1}, \\
\left(R \otimes_{\mathcal{O}} S\right)_{-1} & =R_{1} \otimes_{\mathcal{O}} S_{-1} \oplus R_{-1} \otimes_{\mathcal{O}} S_{1} .
\end{aligned}
$$

Moreover, the wreath product $R \imath S_{w}=R^{\otimes w} * S_{w}$ is $C_{2}$-graded by

$$
\operatorname{deg}\left(r_{1} \otimes \cdots \otimes r_{w}\right) \sigma=\operatorname{sgn}(\sigma) \operatorname{deg} r_{1} \ldots \operatorname{deg} r_{w},
$$

where $r_{1}, \ldots, r_{w} \in R$ are homogeneous elements and $\sigma \in S_{w}$.

By [12] there is a Rickard equivalence between $\mathcal{O}\left(C_{p} \rtimes C_{\frac{p-1}{2}}\right)$ and $B_{0}\left(A_{p}\right)$, which, by [6. Example 5.5], extends to a $C_{2}$-graded equivalence between $\mathcal{O}\left(C_{p} \rtimes C_{p-1}\right)$ and $B_{0}\left(S_{p}\right)$, induced by a complex $X$. Then by [6, Theorem 4.3], the complex $X \imath S_{w}$ induces a Rickard equivalence between $\mathcal{O}\left(\left(C_{p} \rtimes C_{p-1}\right) \curlywedge S_{w}\right)$ and $B_{0}\left(S_{p} \curlywedge S_{w}\right)$. Recall that if $x=x_{i_{1}} \otimes \cdots \otimes x_{i_{w}} \in X^{\otimes w}, x_{i_{j}} \in X_{i_{j}}$ then the action of $\sigma \in S_{w}$ is defined by

$$
{ }^{\sigma} x=(-1)^{\epsilon_{\sigma}\left(i_{1}, \ldots, i_{w}\right)} x_{i_{\sigma^{-1}(1)}} \otimes \cdots \otimes x_{i_{\sigma^{-1}(w)}}
$$

for some $\epsilon_{\sigma}\left(i_{1}, \ldots, i_{w}\right) \in\{0,1\}$. If $r=r_{i_{1}} \otimes \cdots \otimes r_{i_{w}} \in \mathcal{O}\left(C_{p} \rtimes C_{p-1}\right)^{\otimes w}$ and $s=s_{i_{1}} \otimes \cdots \otimes s_{i_{w}} \in B_{0}\left(S_{p}\right)^{\otimes w}$ and $\rho, \sigma, \tau \in S_{w}$, then the multiplication on $X \backslash S_{w}$ is defined by

$$
r \sigma \cdot x \rho \cdot s \tau=\left(r \cdot{ }^{\sigma} x \cdot{ }^{\sigma \rho} s\right) \sigma \tau \rho
$$

Then the above discussion immediately shows that $X \imath S_{w}$ is a complex of $C_{2}$-graded $\left(\mathcal{O}\left(\left(C_{p} \rtimes C_{p-1}\right) \curlywedge S_{w}\right), B_{0}\left(S_{p} \prec S_{w}\right)\right)$-bimodules.

3.6. Next we show that there is a $C_{2}$-graded Morita equivalence between the blocks of defect zero $c_{0} \mathcal{O} S_{t}$ and $f_{0} \mathcal{O} S_{r}$. 
We have that $c_{0} \in \mathcal{O} A_{t}$ and $f_{0} \in \mathcal{O} A_{r}$ since the $p$-cores $\kappa$ and $\rho$ are selfassociated, but these idempotents decompose as $c_{0}=c^{\prime}+c^{\prime \prime}$ and $f_{0}=f^{\prime}+f^{\prime \prime}$ in $\mathcal{O} A_{t}$ and $\mathcal{O} A_{r}$ respectively, where $c^{\prime}, c^{\prime \prime}$, respectively $f^{\prime}, f^{\prime \prime}$ are $C_{2}$-conjugated.

Let $V^{\prime}$ be a $\left(c^{\prime} \mathcal{O} A_{t}, f^{\prime} \mathcal{O} A_{r}\right)$-bimodule inducing a Morita equivalence. We may take $V^{\prime}=U^{\prime} \otimes_{\mathcal{O}} W^{\prime}$, where $U^{\prime}$ is the unique simple left $c^{\prime} \mathcal{O} A_{t}$-module, and $W^{\prime}$ is the unique simple right $f^{\prime} \mathcal{O} A_{r}$-module. Let $V^{\prime \prime}=U^{\prime \prime} \otimes_{\mathcal{O}} W^{\prime \prime}$, where $U^{\prime \prime}$ and $W^{\prime \prime}$ are the $C_{2}$-conjugates of $U^{\prime}$ and $W^{\prime}$ respectively. Then $V:=V^{\prime} \oplus V^{\prime \prime}$ is a $\left(c_{0} \mathcal{O} A_{t} \otimes_{\mathcal{O}}\right.$ $\left.\left(f_{0} \mathcal{O} A_{r}\right)^{\mathrm{op}}\right)$-module, invariant under the conjugation action of $\mathcal{O} S_{t} \otimes_{\mathcal{O}}\left(\mathcal{O} S_{r}\right)^{\mathrm{op}}$. It follows that $V$ extends to the diagonal subalgebra $\Delta=\Delta\left(c_{0} \mathcal{O} S_{t} \otimes_{\mathcal{O}}\left(f_{0} \mathcal{O} S_{r}\right)^{\text {op }}\right)$; hence by [6. Theorem 3.4], $\operatorname{Ind}_{\Delta}^{c_{0} \mathcal{O} S_{t} \otimes \mathcal{O}}\left(f_{0} \mathcal{O} S_{r}\right)^{\mathrm{op}} V$ induces the desired $C_{2}$-graded Morita equivalence.

3.7. It remains to show that there is a $C_{2}$-graded derived equivalence between $b \mathcal{O} S_{n}$ and $e \mathcal{O} S(V)$. Rickard [10] has conjectured that any two blocks of the same weight $w$ of symmetric groups are derived equivalent. He proposed a candidate for a tilting complex which is a generalization of Scopes' Morita equivalence [14]. The conjecture has been recently verified by Chuang and Rouquier [3. Actually, the derived equivalence between $b \mathcal{O} S_{n}$ and $e \mathcal{O} S(V)$ is obtained as a composition of equivalences between blocks forming a so-called $[w: k]$ pair, defined as follows.

Assume that $a \mathcal{O} S_{n}$ is a block of weight $w$ of $\mathcal{O} S_{n}$ corresponding to an abacus whose $j$-th runner has $k$ more beads than the $(j-1)$-th runner. Switching the number of beads on these two runners, we obtain a block $b \mathcal{O} S_{n-k}$ of weight $w$ of $\mathcal{O} S_{n-k}$.

3.7.1. If $k \geq w$, Scopes [14] proved that $a \mathcal{O} S_{n}$ and $b \mathcal{O} S_{n-k}$ are Morita equivalent. We deal with this situation first, since it already uses the main idea. Observe that $M:=a \mathcal{O} S_{n} b$ is an $\left(a \mathcal{O} S_{n}, b \mathcal{O} S_{n-k} \otimes_{\mathcal{O}} \mathcal{O} S_{k}\right)$-bimodule. Then the Morita equivalence is induced by $M \otimes_{\mathcal{O} S_{k}} \mathcal{O}$. One of the problems is that usually $a$ and $b$ are not both self-associated. If $a$ is not self-associated, then $a \mathcal{O} S_{n}$ is isomorphic to $a^{+} \mathcal{O} A_{n}$, where $a^{+}$is the unique block of $\mathcal{O} A_{n}$ covered by $a$. Let $A=a \mathcal{O} S_{n}$ if $a$ is self-associated, and let $A=a^{+} \mathcal{O} A_{n}$ if $a$ is not self-associated. Similarly, let $B=b \mathcal{O} S_{n-k}$ if $b$ is self-associated, and let $B=b^{+} \mathcal{O} A_{n-k}$ if $b$ is not self-associated.

In any case, $A$ and $B$ are $C_{2}$-graded algebras, where if $A=a^{+} \mathcal{O} A_{n}$, then $A_{1}=A$ and $A_{-1}=0$. As in 2.4, we may consider the $\hat{\sigma}$-th conjugate

$$
{ }^{\hat{\sigma}} M=\left(A \otimes_{\mathcal{O}} B^{\mathrm{op}}\right) \hat{\sigma} \simeq \mathcal{O}^{-} \otimes_{\mathcal{O}} M \otimes_{\mathcal{O}} \mathcal{O}^{-}
$$

of $M$, where $\mathcal{O}^{-}$denotes the sign representation. Then ${ }^{\hat{\sigma}} M$ can be identified with $a^{*} \mathcal{O} S_{n} b^{*}$ as $(A, B)$-bimodules. The above discussion shows that ${ }^{\hat{\sigma}} M$ is isomorphic to $M$ as $(A, B)$-bimodules. Since $\hat{C}_{2}$ is cyclic, it follows that $M$ extends to an $\left(A \otimes_{\mathcal{O}} B^{\mathrm{op}}\right) * \hat{C}_{2}$-module, and moreover, $M$ is an $\left(\left(A \otimes_{\mathcal{O}} B^{\mathrm{op}}\right) * \hat{C}_{2}, \mathcal{O} S_{k}\right)$-bimodule. Consequently, $M \otimes_{\mathcal{O} S_{k}} \mathcal{O}$ is an $\left(A \otimes_{\mathcal{O}} B^{\mathrm{op}}\right) * \hat{C}_{2}$-module, hence a $C_{2}$-graded $(A, B)$ bimodule by 2.4 .

3.7.2. For arbitrary $k$, Rickard's complex is a generalization of Scopes' bimodule. We recall its construction following [10] and 2]. Let

$$
r=\max \{i \in \mathbb{N} \mid i(k+i) \leq w\},
$$

and for $0 \leq i \leq r$ let $b_{i}$ be the block of $\mathcal{O} S_{n-k-i}$ having $w-i(k+i)$ and represented by an abacus obtained from the abacus of $b$ by moving $i$ of the beads on the $j$-th 
runner onto the $(j-1)$-th runner. Consider the $\left(a \mathcal{O} S_{n}, b \mathcal{O} S_{n-k}\right)$-bimodule

$$
Y_{i}=a \mathcal{O} S_{n} b_{i} \otimes_{b_{i} \mathcal{O} S_{n-k-i}} b_{i} \mathcal{O} S_{n-k} b .
$$

Using the map

$$
b_{i-1} \mathcal{O} S_{n-k-i+1} b_{i} \otimes_{b_{i} \mathcal{O} S_{n-k-i}} b_{i} \mathcal{O} S_{n-k-i+1} b_{i-1} \rightarrow b_{i} \mathcal{O} S_{n-k-i+1}
$$

induced by multiplication, and the bimodule isomorphisms

$$
\begin{aligned}
a \mathcal{O} S_{n} b_{i-1} \otimes_{b_{i-1} \mathcal{O} S_{n-k-i+1}} b_{i-1} \mathcal{O} S_{n-k-i+1} b_{i} & \simeq a \mathcal{O} S_{n} b_{i} \\
b_{i} \mathcal{O} S_{n-k-i+1} b_{i-1} \otimes_{b_{i-1} \mathcal{O} S_{n-k-i+1}} b_{i-1} \mathcal{O} S_{n-k} b & \simeq b_{i} \mathcal{O} S_{n-k} b
\end{aligned}
$$

one obtains a map $Y_{i} \rightarrow Y_{i-1}$ of $\left(a \mathcal{O} S_{n}, b \mathcal{O}_{n-k}\right)$-bimodules. In order to obtain a complex, the additional structure of these bimodules is needed. Let

$$
X_{i}=\left(a \mathcal{O} S_{n} b_{i} \otimes_{\mathcal{O} S_{k+i}} \mathcal{O}\right) \otimes_{b_{i} \mathcal{O} S_{n-k-i}}\left(\mathcal{O}^{-} \otimes_{\mathcal{O} S_{i}} b_{i} \mathcal{O} S_{n-k} b\right) .
$$

The map $Y_{i} \rightarrow Y_{i-1}$ induces a map $X_{i} \rightarrow X_{i-1}$ simply because $S_{i-1} \leq S_{i}$ and $S_{k+i-1} \leq S_{k+i}$. By [2],

$$
X:=\left(\cdots \rightarrow 0 \rightarrow X_{r} \rightarrow \cdots \rightarrow X_{1} \rightarrow X_{0} \rightarrow 0 \rightarrow \ldots\right)
$$

is a splendid tilting complex of $\left(a \mathcal{O} S_{n}, b \mathcal{O} S_{n-k}\right)$-bimodules.

Define $A$ as in 3.7.1, and similarly let $B_{i}=b_{i} \mathcal{O} S_{n-k-i}$ if $b_{i}$ is self-associated, and $B_{i}=b^{+} \mathcal{O} A_{n-k-i}$ if $b_{i}$ is not self-associated. Observe that $B_{0}=B$. By the procedure described in 3.7.1, $a \mathcal{O} S_{n} b_{i}$ is a $C_{2}$-graded $\left(A, B_{i}\right)$-bimodule and $b_{i} \mathcal{O} S_{n-k} b$ is a $C_{2}$-graded $\left(B_{i}, B\right)$-bimodule. So $Y_{i} \simeq a \mathcal{O} S_{n} b_{i} \otimes_{B_{i}} b_{i} \mathcal{O} S_{n-k} b$, and the maps $Y_{i} \rightarrow Y_{i-1}$ and $X_{i} \rightarrow X_{i-1}$ are $C_{2}$-graded. To see that the map $X_{i} \rightarrow X_{i-1}$ is also $C_{2}$-graded, let

$$
\begin{aligned}
X_{i}^{\prime}:=( & \left.\left(a \mathcal{O} S_{n} b_{i-1} \otimes_{\mathcal{O} S_{k+i-1}} \mathcal{O}\right) \otimes_{B_{i-1}} b_{i-1} \mathcal{O} S_{n-k-i-1} b_{i}\right) \\
\otimes_{B_{i}}\left(b_{i} \mathcal{O} S_{n-k-i+1} b_{i-1}\right. & \left.\otimes_{B_{i-1}}\left(\mathcal{O}^{-} \otimes_{\mathcal{O} S_{i-1}} b_{i-1} \mathcal{O} S_{n-k} b\right)\right) .
\end{aligned}
$$

It is clear that the map $Y_{i} \rightarrow Y_{i-1}$ induces a map $X_{i}^{\prime} \rightarrow X_{i-1}$ of $\left(A \otimes_{\mathcal{O}} B^{\mathrm{op}}\right) * \hat{C}_{2^{-}}$ modules, and the obvious map $X_{i} \rightarrow X_{i}^{\prime}$ is also $\left(A \otimes_{\mathcal{O}} B^{\text {op }}\right) * \hat{C}_{2}$-linear.

3.8. It remains to see that the compatibility with $p^{\prime}$-outer automorphism groups also holds. Fortunately, there is almost nothing to do. With the notation of 3.1 assume that $b^{+}$is the principal block of $\mathcal{O} G^{+}$and that $b$ is the principal block of $\mathcal{O} G$.

Denoting $\tilde{G}=\operatorname{Aut}\left(G^{+}\right)$and $\tilde{H}=N_{\tilde{G}}(D)$, we have that $G \leq \tilde{G}$, and $G=\tilde{G}$ if $n \neq 6$ and $|\tilde{G} / G|=2$ if $n=6$.

Let $n \neq 6$. If $b \neq b^{*}$, then the algebras $b \mathcal{O} G$ and $b^{+} \mathcal{O} G^{+}$are isomorphic, and in this case, the compatibility holds by [6. (5.4)]. If $b=b^{*}=b^{+}$, then the required compatibility just means that there is a $C_{2}$-graded Rickard equivalence between $b \mathcal{O} G$ and $c \mathcal{O} H$, and this is what we have proved above.

Finally, let $n=6$, so that $\left|G^{+}\right|=2^{3} \cdot 3^{2} \cdot 5$. If $p=5$, then there is a $\tilde{G} / G^{+}$. graded Rickard equivalence between $b^{+} \mathcal{O} \tilde{G}$ and $c^{+} \mathcal{O} \tilde{H}$ by [12] and [6. Example 5.5]. If $p=3$, then $D \simeq C_{3} \times C_{3}$. In this case Okuyama constructed in 9 (by using a different method) a Rickard equivalence between $b^{+} \mathcal{O} G^{+}$and $c^{+} \mathcal{O} G^{+}$, and this is compatible with $p^{\prime}$-extensions by [8, Example 3.11]. 


\section{ACKNOWLEDGEMENTS}

This paper was written while the author was visiting the University of Chicago where he was supported by a Fulbright fellowship. He is grateful to J. Alperin, J. Chuang, P. Fong, R. Kessar and J. Rickard for many useful discussions on the subject.

\section{REFERENCES}

1. J. Chuang, The derived categories of some blocks of symmetric groups and a conjecture of Broué, J. Algebra 217 (1999), 114-155. MR 2001a:20024

2. J. Chuang and R. Kessar, Symmetric groups, wreath products, Morita equivalences, and Broué's abelian defect group conjecture, Bull. London Math. Soc. 34 (2002), 174-184. MR 2002m:20016

3. J. Chuang and R. Rouquier, Talks given at the Durham Symposium on Representations of Finite Groups and Related Algebras (1-11 July 2002) .

4. E. C. Dade, Another way to count characters, J. Reine Angew. Math. 510 (1999), 1-55. MR 2000e:20017

5. P. Fong and M. E. Harris, On perfect isometries and isotypies in alternating groups, Trans. Amer. Math. Soc. 349 (1997), 3469-3516. MR 97k:20009

6. A. Marcus, On equivalences between blocks of group algebras: reduction to the simple components, J. Algebra 184 (1996), 372-396. MR 97m:20012

7. A. Marcus, Equivalences induced by graded bimodules, Comm. Algebra 26 (1998), 713-731. MR 99a:16039

8. A. Marcus, Tilting complexes for group graded algebras, J. Group Theory 6 (2003), 175-193.

9. T. Okuyama, Some examples of derived equivalent blocks of finite groups, preprint 1998.

10. J. Rickard, MSRI talk, Berkeley, November 6, 1990.

11. R. Rouquier, Isométries parfaites dans les blocs à défaut abélien des groupes symétriques et sporadiques, J. Algebra 168 (1994), 648-694. MR 95h:20015

12. R. Rouquier, The derived category of blocks with cyclic defect groups, in S. König and A. Zimmermann, Derived Equivalences for Group Rings, Springer-Verlag, Berlin, 1998, pp. 199220.

13. R. Rouquier, Block theory via stable and Rickard equivalences, Modular representation theory of finite groups (Charlottesville, VA, 1998), de Gruyter, Berlin, 2001, pp. 101-146. MR 2003g:20018

14. J. Scopes, Cartan matrices and Morita equivalence for blocks of the symmetric groups, J. Algebra 142 (1991), 441-455. MR 92h:20023

"Babeş-Bolyai" University, Faculty of Mathematics and Computer Science, Str. Mihail Kogălniceanu nR. 1, RO-40084 Cluj-Napoca, Romania

E-mail address: marcus@math.ubbcluj.ro 